\title{
Capture of systemic anticancer therapy use by routinely collected health datasets
} \author{
and David C Currowa \\ a Cancer Institute NSW, Sydney, Australia \\ b Corresponding author: nicola.creighton@health.nsw.gov.au
}

Hanna E Tervonen, Nicola Creighton"a,b, George W Zhao ${ }^{a}$, Megumi Nga

\section{Article history}

Publication date: March 2020

Citation: Tervonen HE, Creighton N, Zhao GW, Ng M, Currow DC. Capture of systemic anticancer therapy use by routinely collected health datasets. Public Health Res Pract. 2020;30(1):e3012004. https://doi. org/10.17061/phrp3012004

\section{Introduction}

There is a growing interest in using person-level linked data to measure the use of systemic anticancer therapies to assess treatment utilisation, disparities in access to treatment and real-world population-level effectiveness. In Australia, anticancer medicines are largely subsidised by the Australian Government under the Pharmaceutical Benefits Scheme (PBS). ${ }^{1,2}$ Administrative processes in hospitals and changing policies may affect the claims data that are available for data linkage. It is not fully understood how complete the capture of anticancer medicine dispensing is in PBS data. Hospital inpatient and Medicare Benefits Schedule (MBS) data also capture some anticancer medicine administration, although information on exact medicines used is not available.

This study examined people undergoing systemic antineoplastic therapy (chemotherapy, targeted therapy) in New South Wales (NSW) public facilities in 2008-2012 to ascertain:

1. Capture of anticancer medicine use in PBS data, and

2. 'Fact of treatment' capture by PBS, MBS and inpatient data combined (i.e., any evidence that anticancer therapy was used).

\section{Methods}

We used NSW Clinical Cancer Registry (2008-2012) (ClinCR) data linked with PBS, NSW Admitted Patient Data Collection (APDC) and MBS data. Linkage was performed by the Centre for Health Record Linkage and Australian Institute of Health and Welfare (AlHW) Data Integration Services Centre. ClinCR data are available for people treated in the majority of NSW public hospitals. ${ }^{3}$ The ClinCR is not a population-based registry. It includes initial episodes of care within 18 months after cancer diagnosis for people treated in public facilities within 12 Local Health Districts in NSW. ${ }^{4}$ Treatment data were collected from source system and medical records and reviewed by local clinical data managers. 
We included people diagnosed with breast (ICD10-AM code C50), colon (C18), head and neck (CO014,C15.3,C30.0,C31.0-31.1,C32-33), lung (C34), neurological $(\mathrm{C} 71, \mathrm{C} 72.8-72.9)$, ovarian $(\mathrm{C} 56, \mathrm{C} 57.0)$ or pancreatic (C25) cancer in NSW in 2008-2012 who underwent systemic antineoplastic therapy (chemotherapy, targeted therapy) within 6 months from diagnosis, as recorded in the ClinCR. These cancer types were selected because they are commonly treated with systemic antineoplastic medicines. Treatment records for hormonal therapies, supportive care medications and clinical trials were excluded. Hormonal therapies were excluded because they are commonly dispensed in a community setting.

The PBS, APDC and MBS data were used to examine whether patients had systemic anticancer therapy records within $+/-60$ days from the ClinCR treatment start date. This time period was selected because it was considered to capture the processing of PBS prescriptions in public hospitals. We included PBS claims for antineoplastic agents (ATC code L01). The APDC procedure and MBS item codes are provided in Appendix 1 (available from: https://figshare.com/articles/Anticancer_medicine_ capture_-_Appendices_docx/11819958). Results were presented as percentage of people with an anticancer record in the ClinCR who had anticancer therapy records in the PBS, APDC, or MBS alone, or in any of the datasets ('fact of treatment').

This study was approved by the NSW Population and Health Services Research Ethics Committee (HREC/15/CIPHS/15) and the AlHW Ethics Committee (EO2016/1/224).

\section{Results}

The PBS data capture was generally high but varied depending on cancer site (Figure 1, Appendix 2, available from: https://figshare.com/articles/Anticancer medicine_capture_-_Appendices_docx/11819958). The highest capture was detected for neurological cancers (92\%) and the lowest capture for head and neck cancers (57\%). For all other cancer sites, capture was $>80 \%$. The MBS capture depended on cancer site (range 12-94\%) and was generally higher than PBS capture, except for colon and neurological cancers. Capture by APDC alone was modest (range 7-33\%). The capture of 'fact of treatment' (PBS, MBS or APDC) was high for all cancers, ranging from $94 \%$ for neurological cancers to $97 \%$ for head and neck cancers.

\section{Discussion}

Capture of 'fact of treatment' by the PBS, MBS and inpatient data for people receiving systemic anticancer therapy in NSW public facilities was high for all cancer sites ( $\geq 94 \%)$. Capture by the PBS, MBS or by inpatient data alone differed by cancer site and likely reflects different treatment options and modes of delivery for each cancer. For example, if oral anticancer medicines are the main treatment, this would be captured by the PBS with limited capture in other data sources since these medicines are usually supplied by community pharmacies.

Figure 1. Capture of anticancer medicine use (\%) compared with NSW ClinCR data (2008-2012) by cancer site and data source

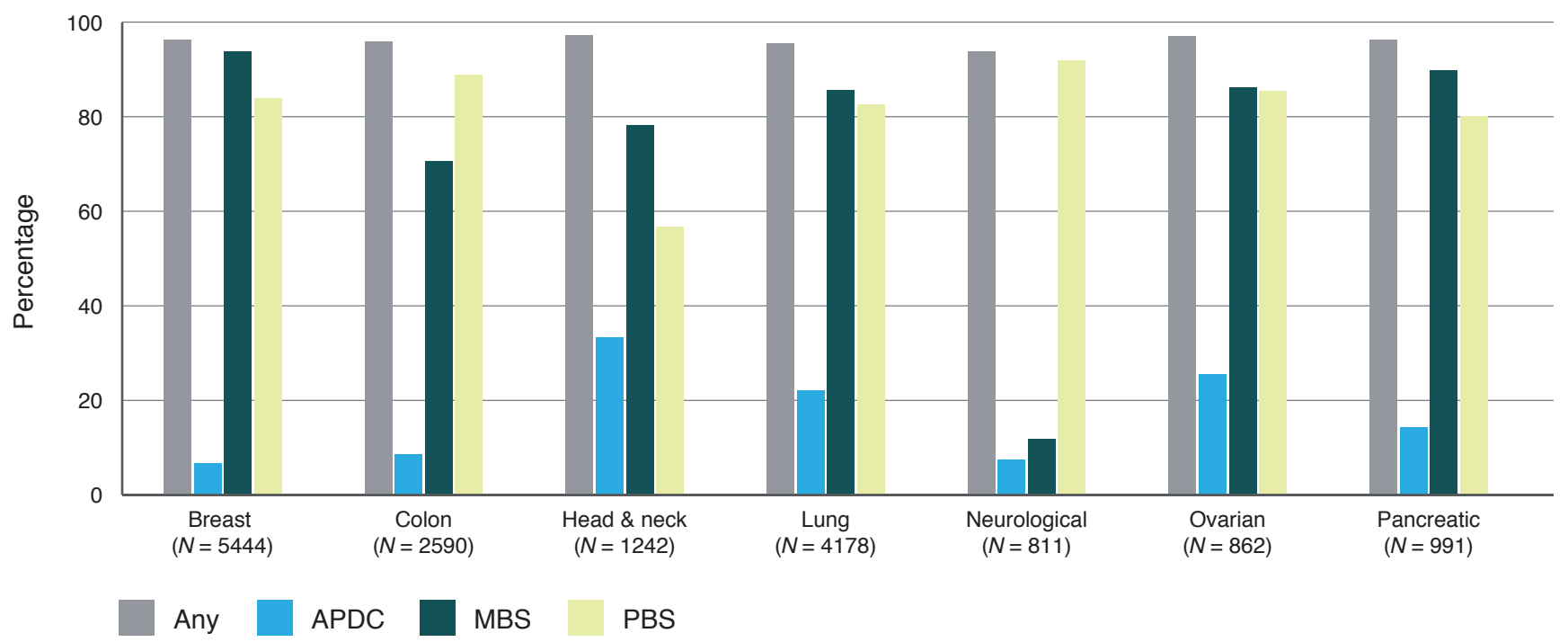

APDC = Admitted Patient Data Collection; ClinCR = NSW Clinical Cancer Registry; MBS = Medicare Benefits Schedule;

PBS = Pharmaceutical Benefits Scheme 
For studies examining specific medicine use, PBS remains the only available routinely collected data source. The PBS capture ranged between 57-92\%. The lowest capture (57\%) was observed for head and neck cancers, which was likely caused by treatments being administered on an admitted basis. Inpatient medicine use in NSW public hospitals is not PBS funded, however systemic anticancer therapy is mostly administered in an ambulatory setting on a non-admitted basis. Hospitals may consider patients as 'privately referred non-admitted' or may undertake in-house compounding for private outpatients to access medicines under PBS arrangements. ${ }^{5}$ Section 100 PBS claims for medicines subsidised under special arrangements have been bulk processed in public hospitals, which would reduce the capture of individual-level data. ${ }^{1}$ The Efficient Funding of Chemotherapy program came into effect in public hospitals in April 2012 and caused a shift to individuallevel processing of some chemotherapy items, increasing PBS capture. Before July 2012, medicines which cost below the PBS patient copayment threshold were not captured in PBS data. However, this is likely to have had only a minor impact on anticancer medicine capture due to their high cost. Medicine use is also not captured in PBS data when medicines are funded by hospitals or privately by patients; are provided by pharmaceutical companies through access programs; are used for non-PBS indications; or are supplied under the Special Access Scheme. ${ }^{6}$

A previous NSW study concluded that PBS data could reliably estimate anticancer medicine use in a sample of women with HER2-positive breast cancer. ${ }^{7}$ To fully understand reasons for incomplete capture of anticancer medicine use by the PBS, a detailed examination of treatments by cancer site will be crucial. Our estimates of PBS capture apply to public hospitals only, as treatment received in the private sector was not recorded in the ClinCR. PBS capture in private hospitals is likely to be higher, as both inpatient and outpatient medicine use in private hospitals is PBS-funded in NSW. ${ }^{1}$ The possibility of false-positive recording of anticancer therapy use in the APDC and MBS is low, because these data record medicine administration. Although the PBS data records dispensing, rather than administration of medicines, the possibility of false-positive recording is low because we only included patients who had received treatment as recorded in the ClinCR.

In conclusion, the capture of anticancer medicine use by the PBS was generally high but varied depending on cancer site. PBS data can be supplemented by MBS and inpatient data, to examine use of systemic anticancer therapy ('fact of treatment') with a very high capture.

\section{Acknowledgements}

We would like to thank the NSW Ministry of Health, the AlHW and the Centre for Health Record Linkage for their assistance with this project. We would also like to thank Alana Little for her valuable help with preparing the manuscript.

\section{Peer review and provenance}

Externally peer reviewed, not commissioned.

\section{Competing interests}

None declared.

\section{Author contributions}

HT, NC and DC developed the study protocol. GZ analysed the data. HT drafted the manuscript. NC, MN and DC provided detailed comments on the paper. All authors contributed to the interpretation of the results and critical revision of the manuscript, and approved the final version of the paper.

\section{References}

1. Mellish L, Karanges EA, Litchfield MJ, Schaffer AL, Blanch B, Daniels BJ, et al. The Australian Pharmaceutical Benefits Scheme data collection: a practical guide for researchers. BMC Res Notes. 2015;8:634.

2. Australian Government Department of Health. Efficient funding of chemotherapy program. Canberra: Commonwealth of Australia; 2018 [cited 2018 Nov 11]. Available from: www.pbs.gov.au/info/browse/section-100/ chemotherapy

3. Cancer Institute NSW. NSW Clinical Cancer Registry (2008-2012). Sydney: NSW Government; 2018 [cited 2018 Nov 11]. Available from: www.cancer.nsw.gov.au/ data-research/data-held-by-cinsw/clinical-cancer-registry

4. Cancer Institute NSW. Whitepaper: CINSW clinical cancer data 2008-2012: an overview and guide for prospective researchers. Sydney: NSW Government CINSW; 2017 [cited 2019 Mar 08]. Available from: www.cancer.nsw. gov.au/getattachment/data-research/data-held-by-cinsw/ clinical-cancer-registry/Whitepaper-CINSW-ClinicalCancer-Data-2008-to-2012.pdf?lang=en-AU

5. Australian Government Department of Health. PBS chemotherapy medicines review. Canberra: Department of Health; 2014 [cited 2018 Nov 11]. Available from: www.health.gov.au/internet/main/publishing.nsf/Content/ chemotherapy-review 
6. Australian Government Department of Health. Therapeutic Good Administration. Special Access Scheme. Canberra: Department of Health; 2019 [cited 2020 Feb 7]. Available from: www.tga.gov.au/form/ special-access-scheme
7. Harris CA, Daniels B, Ward RL, Pearson SA.

Retrospective comparison of Australia's Pharmaceutical Benefits Scheme claims data with prescription data in HER2-positive early breast cancer patients, 2008-2012. Public Health Res Pract. 2017;27(5):e275174.

\section{Copyright: (c)(1)}

(C) 2020 Tervonen et al. This article is licensed under the Creative Commons Attribution-NonCommercial-ShareAlike 4.0 International Licence, which allows others to redistribute, adapt and share this work non-commercially provided they attribute the work and any adapted version of it is distributed under the same Creative Commons licence terms. See: www.creativecommons.org/licenses/by-nc-sa/4.0/ 